\title{
GLObal Robotic telescopes Intelligent Array for e-Science
}

\author{
René Hudec ${ }^{1,2 * \dagger}$ \\ ${ }^{1}$ Czech Technical University in Prague, Faculty of Electrical Engineering, \\ Technicka 2, CZ 16000 Prague, Czech Republic \\ ${ }^{2}$ Astronomical Institute of the Academy of Sciences of the Czech Republic \\ Fricova 298 - CZ 25165 Ondrejov, Czech Republic \\ E-mail: rene.hudec@gmail.com
}

\begin{abstract}
We report here on the ongoing EU FP7 Project GLORIA with emphasis on possibility of investigation of cataclysmic variables by users. GLORIA stands for GLObal Robotic-telescopes Intelligent Array. GLORIA enables the first free and open-access network of robotic telescopes in the world. It represents a Web 2.0 environment where users can do research in astronomy by observing with robotic telescopes, and/or by analyzing data that other users have acquired with GLORIA, or from other free access databases like the European Virtual Observatory.
\end{abstract}

Frontier Research in Astrophysics,

26-31 May 2014

Mondello (Palermo), Italy

\footnotetext{
*Speaker.

† On behalf of large GLORIA project international consortium.
} 


\section{Introduction}

The research in astronomy poses two main challenges, namely 1) the immensity of the sky and 2) the huge amount of astronomical data being gathered. In fact, astronomers are nowadays facing great difficulty in finding the resources to analyze the increasing flood that modern astronomy instruments generate insufficient computing power, insufficiently powerful software tools, and not enough hours in the day. The sky comprises 40000 square degrees (144 million square minutes). The future professional projects (like LSST) intend to observe a very significant fraction of it on a regular basis. In order to meet the above-mentioned challenges, an increasing number of astronomical projects have begun to try to foster citizen participation to help analyze data by using collaborative Internet application (the so-called Web 2.0).

GLORIA stands for "GLObal Robotic-telescopes Intelligent Array". GLORIA represents the first free and open-access network of robotic telescopes in the world. It represents a Web 2.0 environment where users can do research in astronomy by observing with robotic telescopes, and/or by analyzing data that other users have acquired with GLORIA, or from other free access databases like the European Virtual Observatory (http://www.euro-vo.org).

The GLORIA project represents the first attempt to create a network of about 20 robotic telescopes for public access, education, science and much much more. The GLORIA Network has 17 network telescopes now. Few others are expected to join us soon.

Many Internet communities have already formed to speed-up scientific research, to collaborate in documenting something, or for social projects. Research in astronomy can benefit from attracting many eyes to the sky - to detect something in the sky requires looking at the right place at the right time. Our robotic telescopes can search the sky, but the vast quantities of data they produce are far greater than astronomers have time to analyze. GLORIA will provide a way of putting thousands eyes and minds on an astronomy problem. GLORIA is intended to be a Web 2.0 structure, with the possibility of doing real experiments. The community will not only generate content, as in most Web 2.0, but it will control telescopes around the world, both directly and via scheduled observations. The community will take decisions for the network and it will give "intelligence" to GLORIA, while the drudge work (such as drawing up telescope schedules that satisfy various constraints) will be done by algorithms that will be developed for the purpose.

GLORIA project defines free standards, protocols and methodology for the following purposes: controlling Robotic Telescopes and all related instrumentation (i.e. cameras, filter-wheels, domes...); giving Web access to the Network: access to an arbitrary number of robotic telescopes via a web portal; conducting On-line experiments (it will be possible to design specific web environments for controlling telescopes for research on a specific scientific issue; conducting Off-line experiments (it is possible to design specific web environments for analyzing astronomical metadata produced by GLORIA or other databases).

The main part of GLORIA is represented by providing access to robotic telescopes, Online experiments (observations), Offline experiments (research on acquired images), Live broadcasting, Social network + community, and Support. 


\section{Objectives and Benefits of GLORIA Project}

The world-wide network of robotic telescopes (GLORIA) does not intend to compete with LSST, but our underlying idea is that "the more eyes we put on the sky, the more and greater the scientific discoveries will be achieved". Thus, in order to try to improve the way we are doing astronomy research, this project aims to build the first open access world wide network of robotic telescopes to serve citizens from around the world for free, but competing for observing time. Hence GLORIA is indeed an "Intelligent Array" and it bases its intelligence in its community of users (Sanchez Moreno et al. 2013). Like most Web 2.0 projects, GLORIA implements a reputation-based scoring system to reward user contributions, driven by parameters such as the quality of the gathered and processed images, number of hours invested in the observations etc., as well as the votes granted by the rest of the community that finally evaluate the quality of the work done. At the end of month 24 (October 2013) the consortium produced a standard for adding new telescopes and experiments to this network. Unlike other private, profitable ongoing initiatives, the GLORIA network provides a free, twofold service to the community:

- Giving citizens (including professional and amateur astronomers) free access to a network of 17 robotic telescopes spread into 4 continents and both hemispheres. In the case of professional astronomers, preferences will be given to those of developing countries who will lack astronomical facilities in their own nations.

- Giving citizens an easy web access to all the data collected by the robotic telescopes.

Beside giving service to research, in order to manage GLORIA main objective of fostering astronomical research, by allowing near continuous observations of a given target thanks to its worldwide network facilities, the following additional tangible outcomes are pursued:

1. GLORIA enables more telescopes to join, producing methodology, standards, software, and documentation oriented to teach professional and amateurs astronomers to robotize their telescopes and to integrate them into the GLORIA network.

2. GLORIA enables more research goals to be pursued, producing methodology, standards, software, and documentation oriented to teach amateurs and professional astronomers to design new web experiments and to integrate them into the GLORIA network.

3. GLORIA encourages participation in order to increase in number its community of citizen scientists. Newcomers are very welcomed.

4. GLORIA gives free access to knowledge to everybody. All knowledge (software, manual, standards, documents, astronomical images, data, etc) produced by the GLORIA consortium and by the community will have copyleft licenses.

5. GLORIA intends to continue in the future. Since the maintenance of the distributed telescopes is paid by their owners, the cost of the core part is very cheap. The consortium believes that an economic model based in public and private subventions and donations is very possible for a project like this to go on beyond 2014.

\subsection{Impact of the project}

The GLORIA partners really believe in the enormous power of astronomy as a center of interest in scientific and human training of our young people. The children of today will be the 
astronomers of tomorrow. In order to enroll newcomers and awaken interest in astronomy among children, during 2012-14 we have organized the live Internet broadcast of 5 astronomical events: 4 eclipses and a transit of Venus, which was made from the GLORIA network, with associated activities in all schools of the partners countries, with the aim of getting students and teachers participating in research-based science education and improving their motivation to push the barriers of science education further.

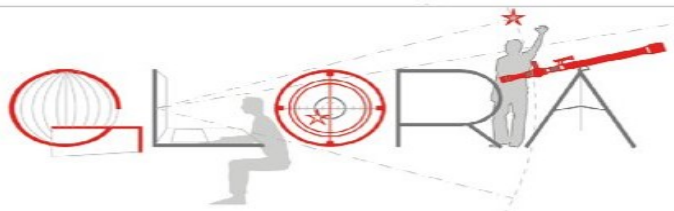

GLObal Robotic telescopes Intelligent Array for e-Science

\begin{tabular}{|l|l|l|l|}
\hline Participant no. & Participant organisation name & $\begin{array}{l}\text { Short } \\
\text { Name }\end{array}$ & Country \\
\hline 1 (Coordinator) & Universidad Politécnica de Madrid & UPM & Spa in \\
\hline 2 & $\begin{array}{l}\text { Astronomical Institute of the Academy of Sciences of the } \\
\text { Czech Republic }\end{array}$ & AUAV & Czech Republic \\
\hline 3 & Consejo Superior de Investigaciones Cientificas & CSIC & Spa in \\
\hline & $\begin{array}{l}\text { Instituto Nacional de Técnica Aeroespacial (Third Pa rty of } \\
\text { participant no 3) }\end{array}$ & INTA & Spain \\
\hline 4 & Czech Technical University & CVUT & Czech Republic \\
\hline 5 & $\begin{array}{l}\text { Institute of Physics of the Academy of Sciences of the } \\
\text { Czech Republic }\end{array}$ & IP-ASCR & Czech Republic \\
\hline 6 & Instituto de Astrofisica de Canarias & IAC & Spain \\
\hline 7 & Istituto Nazionale di Astrofisica & INAF & Italy \\
\hline 8 & $\begin{array}{l}\text { Special Astrophysical Observatory of Russian Academy of } \\
\text { Sciences }\end{array}$ & SAO & Russia \\
\hline 9 & University College Dublin & UCDNUI & Ireland \\
\hline 10 & University of Chile & UC & Chile \\
\hline 11 & Universidad de Málaga & UMA & Spa in \\
\hline 12 & University of Oxford & UOXF & United Kingdom \\
\hline 13 & Uniwersytet Warsawski & UNIWA & Poland \\
\hline
\end{tabular}

Figure 1: The GLORIA project participants

\subsection{The GLORIA Consortium}

GLORIA brings together a critical mass of scientific and technological leaders with extensible expertise in TIC and Astronomical research, dissemination and developed of technological projects. The GLORIA consortium includes these institutions: UPM-Technical University of Madrid Computer Science Faculty-Spain (project coordination); AUAV-Astronomical Institute, Academy of Sciences of the Czech Republic; CSIC-Spanish Research Council-Spain (scientific coordination); CVTU-Czech Technical University-Czech Republic, IP-ASCR-Institute of Physics of the Academy of Sciences of the Czech Republic; IAC-Astrophysic Institute of Canarias-El Teide Observatory-Spain; INAF-Istituto Nazionale di Astrofisica-Italy; SAO-Special Astrophysical Observatory-Russia; UCDNUID-University College Dublin-Ireland; UC-University of ChileChile; UMA-University of Málaga-Spain; UOXF-Oxford University-U.K.; UNIWARSAW-University of Warsaw-Poland.

\section{GLORIA Project and Astrophysics}

Apart from use in citizen science and education, the GLORIA network of robotic telescopes can be used by professional and amateur astronomers to conduct astrophysical research as well. 


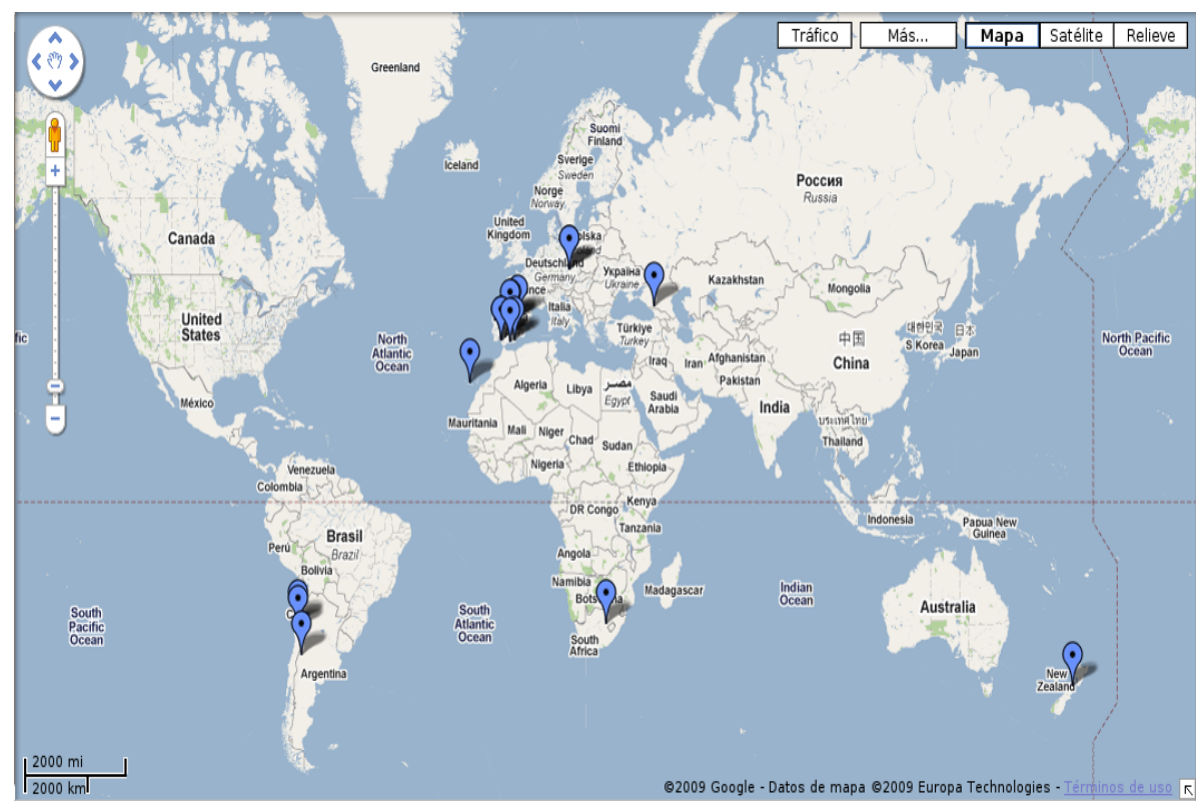

Figure 2: The distribution of the GLORIA project telescopes

The range of use is very wide, below we give just a very few examples.

The CV in Pegasus (found in eruption in May 8, 2010) can serve as an excellent example of a bright object which can be easily detected by amateurs and small cameras and/or telescopes. This object represented a rare type of dwarf nova with very long recurrence time (67 years) but with very large amplitudes (Hudec 2010). We note that the second flare of this CV in 1942 was found using astronomical photographic archives which are perfectly suitable for this kind of work, i.e. searches for past historical flare events (Hudec 1999 and 2007, Hudec et al. 2012). Only very few such objects are known because they can easily escape detection.

In this regard, we also note that the sky contains many yet unknown transients of various kinds. They are identified and attract attention only when they show themselves in their outbursts. The follow-up observations then can lead to the study of their type. One such object detected by a very small telescope is the Liška Flare Star. It is an example of the astronomical discovery of a very rare event with a small amateur telescope (Liška et al. 2014). We suggest that this object is a new flare dMe star of UV Ceti type based on its color, X-ray emission, and the properties of the optical light curve of the flare, identical with the X-ray sources RX J1118.3+1347 and 2E 1115.8+1403. In SIMBAD, these 2 sources are given as different sources but this is probably wrong and it is only one source. The object may be similar to the EXOSAT X-ray source EXO 020528+1454.8 = G 035-027 with a dMe flare star counterpart detected by Hudec et al. (1988).

The investigation of selected cataclysmic variables cas serve as another example (Hudec and Simon, 2015).The observations and research of CVs are expected to be supervised by GLORIA scientists. Everybody can join us at http://gloria-project.eu, and this involves both citizen science and students, as well as professional astronomers. Typically 20 percent of Robotic Telescopes 
(RTs) observing time is expected to be used for GLORIA users, but some telescopes such as BART will provide up to 40 percent.

The analysis with GLORIA telescopes can be effectively supplemented by long-term photometry provided by extended astronomical photographic plate archives. Both databases can provide data for complex analysis of long-term evolution of various astrophysical objects.

Acknowledgments The scientific part of the study (astrophysical aspects) is linked to the the GA CR grant 13-39464J.

\section{References}

[1] Hudec, R., 2010, The Astronomer's Telegram, No. 2619

[2] Hudec, R., et al., 1988, Astronomical Institutes of Czechoslovakia, Bulletin (ISSN 0004-6248), vol. 39 , no. 5 , p. 296-302

[3] Hudec, R., et al., 2012, Acta Polytechnica, IBWS2011 Proceedings, 1(52)

[4] Hudec, R., 2007, in Exploring the Cosmic Frontier: Astrophysical Instruments for the 21st Century. ESO Astrophysics Symposia, ISBN 978-3-540-39755-7. Germany, p.79

[5] Hudec, R., 1999, An introduction to the world's large plate archives, Acta Historica Astronomiae, vol.6, 28

[6] Liška, J., et al., 2015, in preparation.

[7] Sanchez Moreno, F. M., et al., 2013, GLORIA documentation package.

[8] Hudec R. and Simon V., Acta Polytechnica Proc. Series, Vol 2, No 1 (2015): THE GOLDEN AGE OF CATACLYSMIC VARIABLES AND RELATED OBJECTS - II. 